\title{
Preservation of Intuitionistic Fuzzy Preference Relations
}

\author{
Paweł Drygaś \\ Institute of Mathematics, University of Rzeszów, Rejtana 16a, 35-310 Rzeszów, Poland
}

\begin{abstract}
In this paper we consider some properties of intuitionistic fuzzy preference relations. We pay attention to preservation of intuitionistic fuzzy preference relations by aggregation operations. In particular, we know, that a special kind of aggregation operations (lattice operations) do not preserve intuitionistic fuzzy preference relations. We describe the operations which preserve intuitionistic fuzzy preference relations if they are from a special class i.e. they are a decomposable operations.
\end{abstract}

Keywords: intuitionistic fuzzy relations, intuitionistic fuzzy preference relations, aggregation operations, decomposable operations.

\section{Introduction}

We deal with Atanassov's intuitionistic fuzzy relations which were introduced by Atanassov [1] as a generalization of the idea of fuzzy relations defined by Zadeh [17]. Fuzzy sets and relations have many applications in diverse types of areas, for example in data bases, pattern recognition, neural networks, fuzzy modelling, economy, medicine, multicriteria decision making. Moreover, multiattribute decision making using intuitionistic fuzzy sets is possible [10]. We take into account intuitionistic fuzzy preference relations, which are applied in group decision making problems, where the solution from individual preferences over some set of options should be derived. The concept of a preference relation was considered by many authors, in the crisp case for example by [12] and in the fuzzy environment by [4] Szmidt and Kacprzyk [13] generalized the concept of preference from the fuzzy case to the intuitionistic fuzzy case. Next, other autors considered this topic, for example [14] - [16]. In this work we recall some concepts and results useful in our further considerations (section 2). Next, we put some results connected with the preservation of preference relations by aggregation operations (section 3). Finally, we put some open problems connected with intuitionistic preference relations (section 4).

\section{Basic definitions}

Now we recall some definitions which will be helpful in our investigations.

Zadeh (1965) introduced the concept of fuzzy set as follows
Definition 1 (cf. [17]). A fuzzy set $A$ on a universe $X$ is a mapping

$$
A: X \rightarrow[0,1] .
$$

Atanassov intuitionistic fuzzy sets were introduced by Atanassov as an extension of the fuzzy set theory in the following way.

Definition 2 (cf. [1], [2]). An Atanassov intuitionistic fuzzy set $A$ on a universe $X$ is a triple

$$
A=\{(x, \mu(x), \nu(x)): x \in X\},
$$

where

$$
\begin{aligned}
& \mu: X \rightarrow[0,1] \\
& \nu: X \rightarrow[0,1]
\end{aligned}
$$

with the condition

$$
\mu(x)+\nu(x) \leq 1, x \in X .
$$

An Atanassov intuitionistic fuzzy set assigns to each element of the universe not only a membership degree but also a nonmembership degree.

The values $\pi_{A}(x)=1-\mu(x)-\nu(x)$ is called the degree of indeterminacy of $x$ to $A$, or the degree of hesitancy of $x$ to $A$. Especially, if $\pi_{A}(x)=0$ for all $x \in X$, then the intuitionistic fuzzy set $A$ reduced to a fuzzy set.

An Atanassov intuitionistic fuzzy set $A$ on $X$ can be represented by an $L^{*}$-fuzzy set in the sense of Goguen. Namely

Definition 3 (cf. [9]). An L-fuzzy set $A$ on a universe $X$ is a function

$$
A: X \rightarrow L
$$

where $L$ is a lattice.

In this paper we mean by $\left(L^{*}, \leq_{L}\right)$ the following complete lattice

$$
\begin{gathered}
L^{*}=\left\{\left(x_{1}, x_{2}\right) \in[0,1]^{2}: x_{1}+x_{2} \leq 1\right\}, \\
\left(x_{1}, x_{2}\right) \leq L_{L^{*}}\left(y_{1}, y_{2}\right) \Leftrightarrow x_{1} \leq y_{1} \text { and } x_{2} \geq y_{2} .
\end{gathered}
$$

This lattice has the greatest element $1_{L^{*}}=(1,0)$ and the least element $0_{L^{*}}=(0,1)$.

As a special case of sets we may consider relations.

Definition 4 ([17]). Let $X, Y \neq \emptyset$. A fuzzy relation between elements of $X$ and $Y$ is an arbitrary function $R: X \times Y \rightarrow[0,1]$. The family of all fuzzy relations in $X \times Y$ is denoted by $F R(X \times Y)$. In the case $X=Y$ we will use the notation $F R(X)$. 


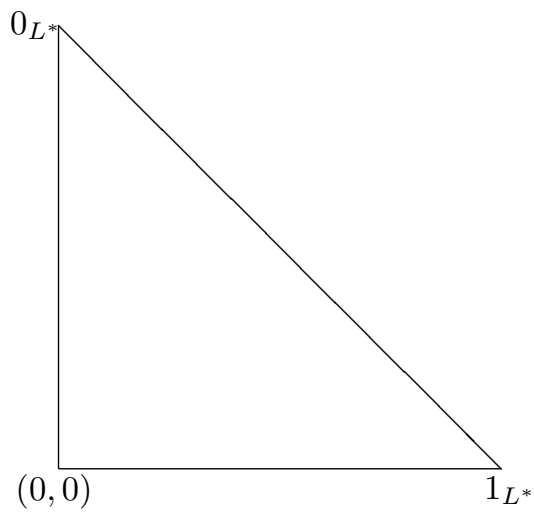

Figure 1: Lattice $L^{*}$

Definition 5 ([1]). Let $X, Y \neq \emptyset, R, R^{d}: X \times Y \rightarrow$ $[0,1]$ be fuzzy relations fulfilling the condition

$$
R(x, y)+R^{d}(x, y) \leq 1,(x, y) \in X \times Y .
$$

A pair $\rho=\left(R, R^{d}\right)$ is called an Atanassov's intuitionistic fuzzy relation. The family of all Atanassov's intuitionistic fuzzy relations described in the given sets $X, Y$ is denoted by $\operatorname{AIFR}(X \times$ $Y)$. In the case $X=Y$ we will use the notation $\operatorname{AIFR}(X)$.

The boundary elements in $\operatorname{AIFR}(X \times Y)$ are $\mathbf{1}=$ $(1,0)$ and $\mathbf{0}=(0,1)$, where 0,1 are the constant fuzzy relations. Basic operations for $\rho=\left(R, R^{d}\right)$, $\sigma=\left(S, S^{d}\right) \in A I F R(X \times Y)$ are the union and the intersection

$$
\begin{aligned}
& \rho \vee \sigma=\left(R \vee S, R^{d} \wedge S^{d}\right), \\
& \rho \wedge \sigma=\left(R \wedge S, R^{d} \vee S^{d}\right) .
\end{aligned}
$$

Similarly, for arbitrary set $T \neq \emptyset$

$$
\begin{aligned}
& \left(\bigvee_{t \in T} \rho_{t}\right)(x, y)=\left(\bigvee_{t \in T} R_{t}(x, y), \bigwedge_{t \in T} R_{t}^{d}(x, y)\right), \\
& \left(\bigwedge_{t \in T} \rho_{t}\right)(x, y)=\left(\bigwedge_{t \in T} R_{t}(x, y), \bigvee_{t \in T} R_{t}^{d}(x, y)\right) .
\end{aligned}
$$

Moreover, the order in $A I F S(X \times Y)$ is defined as follows

$$
\rho \leq \sigma \Leftrightarrow\left(R \leq S, S^{d} \leq R^{d}\right) .
$$

The pair $(A I F R(X \times Y), \leq)$ is a partially ordered set. Operations $\vee, \wedge$ are the binary supremum and infimum in the family $\operatorname{AIFR}(X \times Y)$, respectively. The family $(A I F R(X \times Y), \vee, \wedge)$ is a complete, distributive lattice.

If we compare the above and the definition 3 and take into account the condition (1), then fuzzy relation can be seen not as a pair of relations, but as a relation with values of the lattice $L^{*}$.

The fuzzy relation $\pi_{\rho}: X \times Y \rightarrow[0,1]$ is associated with each Atanassov's intuitionistic fuzzy relation $\rho=\left(R, R^{d}\right)$, where

$$
\pi_{\rho}(x, y)=1-R(x, y)-R^{d}(x, y), x \in X, y \in Y .
$$

The number $\pi_{\rho}(x, y)$ is called an index of an element $(x, y)$ in an Atanassov's intuitionistic fuzzy relation $\rho$. It is also described as an index (a degree) of hesitation whether $x$ and $y$ are in the relation $\rho$ or not. This value is also regarded as a measure of nondeterminacy or uncertainty (see [11]) and is useful in applications. Intuitionistic fuzzy indices allow to calculate the best final result and the worst one that may be expected in a process leading to a final optimal decision (see [11]).

Let us recall the notion of the composition

Definition 6 ([9], [3]). Let $\sigma=\left(S, S^{d}\right) \in$ $A I F R(X \times Y), \rho=\left(R, R^{d}\right) \in A I F R(Y \times Z)$. By the composition of relations $\sigma$ and $\rho$ we call the relation $\sigma \circ \rho \in A I F R(X \times Z)$,

$$
(\sigma \circ \rho)(x, z)=\left((S \circ R)(x, z),\left(S^{d} \circ^{\prime} R^{d}\right)(x, z)\right),
$$

where

$$
\begin{aligned}
(S \circ R)(x, z) & =\bigvee_{y \in Y}(S(x, y) \wedge R(y, z)), \\
\left(S^{d} \circ^{\prime} R^{d}\right)(x, z) & =\bigwedge_{y \in Y}\left(S^{d}(x, y) \vee R^{d}(y, z)\right)
\end{aligned}
$$

If we consider decision making problems in the intuitionistic fuzzy environment we deal with the finite set of alternatives $X=\left\{x_{1}, \ldots, x_{n}\right\}$ and an expert who needs to provide his preference information over alternatives. In the sequel, we will consider a preference relation on a finite set $X=$ $\left\{x_{1}, \ldots, x_{n}\right\}$. In this situation intuitionistic fuzzy relations may be represented by matrices.

Definition 7 ([15], [13]). Let card $X=n$. An intuitionistic fuzzy preference relation $\rho$ on the set $X$ is represented by a matrix $\rho=\left(\rho_{i j}\right)_{n \times n}$ with $\rho_{i j}=\left(R(i, j), R^{d}(i, j)\right)$, for all $i, j=1, \ldots, n$, where $\rho_{i j}$ is an intuitionistic fuzzy value, composed by the degree $R(i, j)$ to which $x_{i}$ is preferred to $x_{j}$, the degree $R^{d}(i, j)$ to which $x_{i}$ is non-preferred to $x_{j}$. Furthermore, for all $i, j=1, \ldots, n$, the values $R(i, j)$, $R^{d}(i, j)$ satisfy for all $i, j=1, \ldots, n$ the following properties:

$$
\begin{gathered}
0 \leq R(i, j)+R^{d}(i, j) \leq 1, \\
R(i, j)=R^{d}(j, i), R(j, i)=R^{d}(i, j), \\
R(i, i)=R^{d}(i, i)=0.5 .
\end{gathered}
$$

Moreover, by $\pi_{\rho}(i, j)$ we denote the uncertainty degree to which $x_{i}$ is preferred to $x_{j}$.

Remark 1. Directly from this definition it follows that for all $i, j=1, \ldots, n$ we have:

1. $\pi_{\rho}(i, j)=\pi_{\rho}(j, i)$.

2. In the definition it is enough to assume one of these conditions:

$$
\begin{aligned}
& R(i, j)=R^{d}(j, i), \\
& R(j, i)=R^{d}(i, j) .
\end{aligned}
$$

3. In the definition it is enough to assume one of these conditions:

$R(i, i)=0.5$

$R^{d}(i, i)=0.5$. 


\section{Aggregation operations and intuitionistic fuzzy preference relations}

In this section we consider the preservation of intuitionistic fuzzy preference relations by aggregation operations. In particular lattice operations in the family $\operatorname{AIFR}(\mathrm{X})$ do not preserve an intuitionistic fuzzy preference relation, i.e. if $\rho$ and $\sigma$ are intuitionistic fuzzy preference relations, then their sum and intersection need not have this property.

Example $1([8])$. Let card $X=2$ and $\rho=\left(R, R^{d}\right)$, $\sigma=\left(S, S^{d}\right) \in A I F R(X)$ be intuitionistic fuzzy preference relations represented by the matrices:

$$
\begin{aligned}
& \rho=\left[\begin{array}{ll}
(0.5,0.5) & (0.3,0.6) \\
(0.6,0.3) & (0.5,0.5)
\end{array}\right], \\
& \sigma=\left[\begin{array}{ll}
(0.5,0.5) & (1,0) \\
(0,1) & (0.5,0.5)
\end{array}\right] .
\end{aligned}
$$

Then according to (2), we obtain

$$
\begin{aligned}
& \rho \vee \sigma=\left[\begin{array}{ll}
(0.5,0.5) & (1,0) \\
(0.6,0.3) & (0.5,0.5)
\end{array}\right], \\
& \rho \wedge \sigma=\left[\begin{array}{ll}
(0.5,0.5) & (0.3,0.6) \\
(0,1) & (0.5,0.5)
\end{array}\right] .
\end{aligned}
$$

We see that none of the relations $\rho \vee \sigma, \rho \wedge \sigma$ is a preference relation.

The lattice operations are a special case of aggregation operations. So, we ask when using aggregation operations instead lattice operations the intuitionistic fuzzy preference relations will be preserved.

Definition 8. Let $A:[0,1]^{2} \rightarrow[0,1]$ be an binary operation and $\rho, \sigma \in A I F R(X)$. By aggregation fuzzy relation we call $\varrho \in A I F R(X)$,

$$
\varrho(x, y)=A(\rho(x, y), \sigma(x, y)), x, y \in X .
$$

If we use aggregation operations composed by arithmetic mean we obtain

\section{Example 2. Let}

$$
A(x, y)=\left(\frac{x_{1}+y_{1}}{2}, \frac{x_{2}+y_{2}}{2}\right)
$$

and $\rho=\left(R, R^{d}\right), \sigma=\left(S, S^{d}\right) \in A I F R(X)$ be an intuitionistic fuzzy preference relations. Then

$$
\begin{gathered}
\varrho_{i j}=\left(P(i, j), P^{d}(i, j)\right)= \\
A\left(\left(R(i, j), R^{d}(i, j)\right),\left(S(i, j), S^{d}(i, j)\right)\right)= \\
\left(\frac{R(i, j)+S(i, j)}{2}, \frac{R^{d}(i, j)+S^{d}(i, j)}{2}\right) .
\end{gathered}
$$

Since

$$
\begin{aligned}
& 0 \leq R(i, j)+R^{d}(i, j) \leq 1 \\
& 0 \leq S(i, j)+S^{d}(i, j) \leq 1
\end{aligned}
$$

then

$$
0 \leq \frac{R(i, j)+S(i, j)}{2}+\frac{R^{d}(i, j)+S^{d}(i, j)}{2} \leq 1 .
$$

So, we have (6). Moreover, we have

$$
\begin{array}{r}
R(i, j)=R^{d}(j, i), \\
S(i, j)=S^{d}(j, i)
\end{array}
$$

and directly we obtain

$P(i, j)=\frac{R(i, j)+S(i, j)}{2}=\frac{\left.R^{(} j, i\right)+S^{d}(j, i)}{2}=P^{d}(j, i)$

which together with Remark 1 gives (7). By simple accounts we obtain

$$
P(i, i)=\frac{R(i, i)+S(i, i)}{2}=\frac{0.5+0.5}{2}=0.5
$$

Which means that aggregation A preserved intuitionistic fuzzy preference relations.

Now we ask about properties of aggregation operations which leads to preservation of intuitionistic fuzzy preference relations. Firstly we examine the decomposable operations

Definition 9 ([7], cf. [6]). An operation $A$ : $\left(L^{*}\right)^{2} \rightarrow L^{*}$ is called decomposable if there exist operations $A_{1}, A_{2}:[0,1]^{2} \rightarrow[0,1]$ such that for all $x, y \in L^{*}$

$$
A(x, y)=\left(A_{1}\left(x_{1}, y_{1}\right), A_{2}\left(x_{2}, y_{2}\right)\right)
$$

where $x=\left(x_{1}, x_{2}\right), y=\left(y_{1}, y_{2}\right)$.

The following Lemma characterize some family of the above operations

Lemma 1 (c.f. [6]). Increasing operations $A_{1}, A_{2}$ : $[0,1]^{2} \rightarrow[0,1]$ lead to the decomposable operation $A$ if and only if $A_{1} \leq A_{2}^{\prime}$, where $A_{2}^{\prime}$ is a dual operation to $A_{2}$, i.e. $A_{2}^{\prime}(x, y)=1-A_{2}(1-x, 1-y)$ for all $x, y \in[0,1]$.

One example of decomposable operations are triangular norms and conorms. More information about these operations can be found in [6].

Theorem 1. Let $A: L^{* 2} \rightarrow L^{*}$ be a decomposable operation. Aggregation of intuitionistic fuzzy preference relations is a intuitionistic fuzzy preference relation if and only if $A_{1}=A_{2}$ and $A_{1}(0.5,0.5)=0.5$.

Proof. Let $A=\left(A_{1}, A_{2}\right)$ be a decomposable operation, $x, y \in[0,1]$ and $\rho=\left(R, R^{d}\right)$ and $\sigma=\left(S, S^{d}\right)$ be such intuitionistic fuzzy preference relations that $R(i, j)=x, S(i, j)=y$ for $i \leq j, \varrho=A(\rho, \sigma)=$ $\left(P(i, j), P^{d}(i, j)\right)$ be an intuitonistic fuzzy relation. If $\varrho$ is an intuitionistic fuzzy preference relation, then

$$
A\left(\left(R(i, j), R^{d}(i, j)\right),\left(S(i, j), S^{d}(i, j)\right)\right)=
$$




$$
\left(A_{1}(R(i, j), S(i, j)), A_{2}\left(R^{d}(i, j), S^{d}(i, j)\right)\right) .
$$

Since

$$
\begin{aligned}
R(i, j) & =R^{d}(j, i), \\
S(i, j) & =S^{d}(j, i), \\
A_{1}(R(i, j), S(i, j)) & =A_{2}\left(R^{d}(j, i), S^{d}(j, i)\right)
\end{aligned}
$$

then

$$
A_{1}(R(i, j), S(i, j))=A_{2}((R(i, j), S(i, j)),
$$

i.e.

$$
A_{1}(x, y)=A_{2}(x, y)
$$

which means that $A_{1}=A_{2}$. Moreover, we have

$$
\begin{aligned}
R(i, i) & =R^{d}(i, i)=0.5, \\
S(i, i) & =S^{d}(i, i)=0.5 \\
A_{1}(R(i, i), S(i, i)) & =A_{2}\left(R^{d}(i, i), S^{d}(i, i)\right)=0.5
\end{aligned}
$$

which gives

$$
A_{1}(0.5,0.5)=0.5 \text {. }
$$

Let now $A_{1}=A_{2}$ and 0.5 be an idempotent element of $A_{1}$, i.e. $A_{1}(0.5,0.5)=0.5$. Since $A$ is a decomposable operation, then by above and Lemma 1 we have

$$
A_{1}(x, y) \leq 1-A_{1}(1-x, 1-y)
$$

and by simple computation

$$
0 \leq T(i, j)+T^{d}(i, j) \leq 1 .
$$

So, we have (6). To prove (7) we have

$$
\begin{aligned}
T(i, j) & =A_{1}(R(i, j), S(i, j)) \\
& =A_{1}\left(R^{d}(j, i), S^{d}(j, i)\right) \\
& =A_{2}\left(R^{d}(j, i), S^{d}(j, i)\right) \\
& =T^{d}(j, i) .
\end{aligned}
$$

Using Remark 1 we have (7).

Moreover, we have

$$
\begin{aligned}
& R(i, i)=R^{d}(i, i)=0.5, \\
& S(i, i)=S^{d}(i, i)=0.5,
\end{aligned}
$$

which together with idempotency of $A_{1}$ at point 0.5 gives

$$
\begin{gathered}
T(i, i)=A_{1}(R(i, i), S(i, i))=A_{1}(0.5,0.5)=0.5 \\
T^{d}(i, i)=A_{2}\left(R^{d}(i, i), S^{d}(i, i)\right) \\
=A_{1}\left(R^{d}(i, i), S^{d}(i, i)\right)=A_{1}(0.5,0.5)=0.5 .
\end{gathered}
$$

Corollary 1. There is no decomposable triangular norm or triangular conorm which preserved intuitionistic fuzzy preference relations.
Corollary 2. Since the lattice operations are special cases of triangular norms and triangular conorms, then they do not preserve intuitionistic fuzzy preference relations.

As a special case of aggregation operations we may consider the weighted arithmetic mean. Then we obtain

Corollary 3 (cf. [16], Theorem 1). Let $\rho, \sigma$ be intuitionistic fuzzy preference relations given by the experts $e_{1}, e_{2}$, and $\lambda_{1}, \lambda_{2}$ be the weight values of experts, where $\rho=\left(R, R^{d}\right), \sigma=\left(S, S^{d}\right), \lambda_{1}+\lambda_{2}=$ 1. Then the aggregation $\varrho=\left(P, P^{d}\right)$ of $\rho, \sigma$ is also an intuitionistic fuzzy preference relation, where

$$
\begin{gathered}
P(i, j)=\lambda_{1} R(i, j)+\lambda_{2} S(i, j), \\
P^{d}(i, j)=\lambda_{1} R^{d}(i, j)+\lambda_{2} S^{d}(i, j) .
\end{gathered}
$$

Proof. The operation which we use fulfill the assumption of Theorem 1, i.e. it is decomposable operation with the weighted arithmetic mean as components.

In a similar way we investigate whether the intuitionistic fuzzy preference relations are preserved by pseudo-t-representable operations.

Open Problem 1. In the above considerations, we presented the conditions to preserve intuitionistic fuzzy preference relations by decomposable operations. The open problem is to find the conditions for arbitrary aggregation operation to allow us to preserve intuitionistic fuzzy preference relations.

\section{Composition of intuitionistic fuzzy preference relations}

In this section we consider the preservation of composition of intuitionistic fuzzy preference relations. The composition in the family $\operatorname{AIFR}(\mathrm{X})$ does not preserve a intuitionistic fuzzy preference relations, i.e. if $\rho$ and $\sigma$ are intuitionistic preference relations, then their composition need not have this property.

Example 3. Let card $X=2$ and $\rho=\left(R, R^{d}\right)$, $\sigma=\left(S, S^{d}\right) \in \operatorname{AIFR}(X)$ be intuitionistic fuzzy preference relations defined in Example 1.

Then according to (4) and (5), we obtain

$$
\begin{aligned}
& \rho \circ \sigma=\left[\begin{array}{ll}
(0.5,0.5) & (0.5,0.5) \\
(0.5,0.5) & (0.6,0.3)
\end{array}\right], \\
& \rho \circ \rho=\left[\begin{array}{ll}
(0.5,0.5) & (0.3,0.6) \\
(0.5,0.5) & (0.5,0.5)
\end{array}\right] .
\end{aligned}
$$

We see that none of the relations $\rho \circ \sigma \rho \circ \rho$ is a intuitionistic fuzzy preference relation.

Open Problem 2. As can be seen in the above example the composition of intuitionistic fuzzy relations do not preserve intutionistic fuzzy preference relations. So, we ask about the additional assumption for intutionistic fuzzy preference relations allow us to obtain intuitionistic fuzzy preference relation as composition. 
Acknowledgments.

This paper is partially supported by the Ministry of Science and Higher Education Grant Nr N N519 384936 .

\section{References}

[1] K.T. Atanassov, Intuitionistic fuzzy sets, Fuzzy Sets Syst. 20 (1986), 87-96. 15

[2] K.T. Atanassov, Intuitionistic Fuzzy Sets: Theory and Applications. Springer-Verlag, 1999.

[3] P. Burillo, H. Bustince, Intuitionistic Fuzzy Relations. Effect of Atanassov's Operators on the Properties of the Intuitionistic Fuzzy Relations, Math. Soft Comp. 2 (1995), 117-148.

[4] F. Chiclana, E. Herrera-Viedma, S. Alonso, R. A. M. Pereira, Preferences and consistency issues in group decision making, in: H. Bustince at al. (Eds.), Fuzzy Sets and Their Extensions: Representation, Aggregation and Models, Springer-Verlag, Berlin, 2008, 219-237.

[5] G. Deschrijver, E.E. Kerre, On the composition of intuitionistic fuzzy relations, Fuzzy Sets Syst. 136(3) (2003), 333-361.

[6] G. Deschrijver, C. Cornelis, E.E. Kerre On the Representation of Intuitonistic Fuzzy $t$-Norms and $t$-Conorms. IEEE Transactions on Fuzzy Syst. 12, (2004), 45-61.

[7] P. Drygaś, The problem of distributivity between binary operations in bifuzzy set theory, in: Proceedings of IPMU'08, L. Magdalena, M. Ojeda-Aciego, J.L. Verdegay (eds), pp. 16481653, Torremolinos (Malaga) 2008.

[8] U. Dudziak, B. Pȩkala, Properties of intuitionistic fuzzy preference relations, (submited to SRI PAS IBS PAN) 2010.

[9] A. Goguen, L-fuzzy sets, J. Math. Anal. Appl., 18 (1967), 145-174.

[10] D.-F. Li, Multiattribute decision making models and method using intuitionistic fuzzy sets, J. Comp. Syst. Sci. 70 (2005), 73-85.

[11] L. Lin, X-H. Yuan and Z-Q. Xia, Multicriteria fuzzy decision-making methods based on intuitionistic fuzzy sets, J. Comp. Syst. Sci., 73 (2007), 84-88.

[12] M. Roubens, P. Vincke, Preference Modelling, Springer-Verlag, Berlin, 1985.

[13] E. Szmidt, J. Kacprzyk, Using intuitionistic fuzzy sets in group decision making, Control Cybernet. 31 (2002), 1037-1053.

[14] E. Szmidt, J. Kacprzyk, Atanassov's Intuitionistic Fuzzy Sets as a Promising Tool for Extended Fuzzy Decision Making Models, in: H. Bustince at al. (Eds.), Fuzzy Sets and Their Extensions: Representation, Aggregation and Models, Springer-Verlag, Berlin, 2008, 335-355.

[15] Z. Xu, Intuitionistic preference relations and their application in group decision making, Inform. Sci. 177 (2007), 2363-2379.
[16] Z. Xu, R. R. Yager, Intuitionistic and intervalvalued intuitionistic fuzzy preference relations and their measures of similarity for the evaluation of agreement within a group, Fuzzy Optim. Decis. Mak. 8 (2009), 123-139.

[17] L.A. Zadeh, Fuzzy sets, Inform. Control 8 (1965), 338-353. 